International Mathematical Forum, 1, 2006, no. 38, 1881-1885

\title{
A Note on Operators in Hilbert $\mathrm{C}^{*}$-Modules
}

\author{
M. Khanehgir and M. Hassani \\ Dept. of Math., Islamic Azad University of Mashhad \\ Mashhad P.O. Box 413-91735, Iran \\ khanehgir@mshdiau.ac.ir \\ hassani@mshdiau.ac.ir
}

\begin{abstract}
We explore commutativity up to a unitary factor for the pair of self-adjoint operators in Hilbert $C^{*}$-modules.
\end{abstract}

Mathematics Subject Classification: 46H40, 46L57

Keywords: Hilbert $C^{*}$-modules, partial isometry, unitary map

\section{Introduction}

Let $A$ be a $C^{*}$-algebra (not necessarily unital or commutative). An inner product $A$-module is a linear space $E$ which is right $A$-module (with compatible scalar multiplication: $\lambda(x a)=(\lambda x) a=x(\lambda a)$ for $x \in E, a \in A, \lambda \in \mathcal{C})$, together with a map $(x, y) \rightarrow\langle x, y\rangle: E \times E \rightarrow A$ such that

(i) $\langle x, \alpha y+\beta z\rangle=\alpha\langle x, y\rangle+\beta\langle x, z\rangle$

(ii) $\langle x, y a\rangle=\langle x, y\rangle a \quad(x, y \in E, a \in A)$

(iii) $\langle x, y\rangle^{*}=\langle y, x\rangle \quad(x, y \in E)$

(iv) $\langle x, x\rangle \geq 0$ if $\langle x, x\rangle=0$ then $x=0$.

Given Hilbert $C^{*}$-modules $E$ and $F$, denoted by $L(E, F)$ the set of all (bounded) adjointable maps. Also $K(E, F)$ is the closed linear subspace 
spanned by $\left\{\Theta_{x, y}: x \in F, y \in E\right\}$ in $L(E, F)$, where $\Theta_{x, y}(z)=x<y, z>$, for each $z \in E$. Specially, if $E=F$, we write $L(E), K(E)$ respectively.

It is well known that $L(E)$ is a $C^{*}$-algebra and $K(E)$ is the closed two-sided ideal in $L(E)$. The basic materials can see [1].

If $E$ is a Hilbert $C^{*}$-module, $x, y$ in $E$ is said to be orthogonal if $\langle x, y\rangle=0$. In this case, denote by $x \perp y$. Given a closed submodule $F$ of $E$, set $F^{\perp}=$ $\{y \in E:\langle x, y\rangle=0, \forall x \in F\}$, then $F^{\perp}$ is called orthogonal complement of $F$. Furthermore, if $F \oplus F^{\perp}=E$ then $F$ is said to be complemented submodule. We know that a closed submodule of a Hilbert $C^{*}$-module need not be complemented.

The following proposition gives an equivalent characterization of complemented closed submodule.

Proposition 1.1: Let $A$ be a $C^{*}$-algebra, $E$ is a Hilbert $C^{*}$-module, $E_{0}$ is a closed submodule in $E$. The following are equivalent:

(1) $E_{0}$ is a complemented submodule.

(2) there is a projection $p$ in $L(E)$ such that $K\left(E_{0}\right) \cong p K(E) p$, where $R(p)$ is the range of $p$.

Proof: See [2].

If $F$ is complemented then for each $z$ in $E$ we can uniquely write $z=x+y$ with $x$ in $F$ and $y$ in $F^{\perp}$. Just as in the case of Hilbert spaces, the equation $x=P z$ defines a projection $P$ in $L(E)$ whose range is $F$. Conversely, if $P$ is a projection in $L(E)$ then the range of $P$ is a complemented submodule of $E$, since it is easy to check that $\operatorname{ran}(P)^{\perp}=\operatorname{ran}(1-P)=\operatorname{ker}(P)$.

An operator $u \in L(E, F)$ is said to be unitary if $u^{*} u=1_{E}, \quad u u^{*}=1_{F}$. Also if $t \in L(E)$, then we define spectrum of $t$ is the set $\operatorname{sp}(t)=\{\lambda \in \mathcal{C}: \lambda I-t$ is not invertible $\}$ and we say $t$ is positive and we denoted it by $t \in L(E)^{+}$if $s p(t) \subseteq \mathbb{R}^{+}$.

The theorem of Miscenko which enables one to conclude that certain submodules are complemented.

Theorem 1.2: Let $E, F$ be Hilbert $A$-modules and suppose that $t$ in $L(E, F)$ has closed range. then

(i) $\operatorname{ker}(t)$ is complemented submodule of $E$ and $\operatorname{ker}(t)^{\perp}=\operatorname{ran}\left(t^{*}\right)$. 
(ii) $\operatorname{ran}(t)$ is complemented submodule of $F$ and $\operatorname{ran}(t)^{\perp}=\operatorname{ker}\left(t^{*}\right)$.

(iii) the mapping $t^{*} \in L(F, E)$ also has closed range.

Proof: See [1].

In the following we give some useful theorems which we need them.

Theorem 1.3: Let $u$ be a linear map from $E$ to $F$. Then the following condition are equivalent:

(i) $u$ is an isometric, surjective $A$-linear map.

(ii) $u$ is a unitary element of $L(E, F)$.

Proof: See [1].

We know that if $u$ is isometric then $|u(x)|=|x|$ for each $x \in E$.

Theorem 1.4: For $t$ in $L(E, F), t^{*} F$ and $t^{*} t E$ have the same closures.

Proof: See [1].

Theorem 1.5: If $t \in L(E)^{+}$has closed range then $\operatorname{ran}(t)=\operatorname{ran}\left(t^{\frac{1}{2}}\right)$.

Proof: Trivially $\operatorname{ran}(t) \subseteq \operatorname{ran}\left(t^{\frac{1}{2}}\right)$. Conversely consider $C[z]$ denotes the algebra of all polynomials in an indeterminate $z$ with complex coefficients and suppose that $A$ is unital algebra. We know that the map from $C[z]$ into $A$ which takes $P$ to $P(a)$ which is $a \in A$, is a unital homomorphism. By StoneWeierstrass theorem (See [3]) there is a sequence of polynomials with zero constant coefficient which tends to continuous function $\sqrt{x}$. Now we obtain $\operatorname{ran}\left(t^{\frac{1}{2}}\right) \subseteq \operatorname{ran}(t)$

Definition 1.6: An element $C$ in $L(E, F)$ is called a partial isometry (from $E_{0}$ to $\left.F_{0}\right)$ if $F_{0}=\operatorname{ran}(C)$ is complemented in $F$ and there exists a complemented submodule $E_{0}$ of $E$ such that $C$ is isometric from $E_{0}$ onto $F_{0}$ and $C\left(E_{0}^{\perp}\right)=\{0\}$.

Suppose $t \in L(E, F)$ and that the closures of the ranges of $t$ and $t^{*}$ are both complemented, then define $u: \operatorname{ran}(|t|) \rightarrow \operatorname{ran}(t)$ by $u(|t| x)=t x$. It is easily seen that $u$ is isometric. We define $u$ on $\overline{\operatorname{ran}(|t|)}$ as follows.

For $x \in \overline{\operatorname{ran}(|t|)}$ there is a sequence $\left\{a_{n}\right\}_{n \in N} \subseteq \operatorname{ran}(|t|)$ in which $a_{n} \rightarrow x$ as $n$ tends to infinity. The sequence $\left\{u\left(a_{n}\right)\right\}_{n \in N}$ is a Cauchy sequence in $E$, so converges to an element of $\overline{\operatorname{ran}(t)}$ say $a$. Now we define $u(x)=a$. Also we define $u$ is equal zero on $\overline{\operatorname{ran}(|t|)}^{\perp}$. Evidently $t=u|t|$ and $u$ is a partial 
isometry, so $t$ has polar decomposition.

Just as for Hilbert space, one can easily that the following theorem holds for Hilbert $C^{*}$-modules.

Theorem 1.7: For an element $C$ of $L(E, F)$ the following condition are equivalent.

(i) $C$ is a partial isometry.

(ii) $C^{*} C$ is a projection in $L(E)$.

(iii) $C C^{*}$ is a projection in $L(F)$.

(iv) $C C^{*} C=C$.

(v) $C^{*} C C^{*}=C^{*}$.

Proof: See [1].

Lemma 1.8: If $t \in L(E)$ is normal then $\operatorname{ker}(t)=\operatorname{ker}\left(t^{*}\right)$.

Proof: We have

$$
\left\langle t^{*} t x-t t^{*} x, x\right\rangle=|t x|^{2}-\left|t^{*} x\right|^{2}
$$

So $t$ is normal iff $|t x|=\left|t^{*} x\right|$ and therefore $\operatorname{ker}(t)=\operatorname{ker}\left(t^{*}\right)$.

\section{Main Theorem}

Theorem: Let $t, s$ be bounded self-adjoint operators on Hilbert $C^{*}$-module $E$ and $t s$ and $|t s|$ have closed range. The following are equivalent:

(i) $t s^{2} t=s t^{2} s$.

(ii) $t s=$ ust for some unitary $u$.

Proof: $(i i) \rightarrow(i):$ Observe that $s t=t s u^{*}$, and so $t s=u t s u^{*}$. Hence $t s$ commutes with $u$ and $u^{*}$, and similarly for st. Thus we get

$$
t s^{2} t=u s t u^{*} t s=s t u u^{*} t s=s t^{2} s .
$$

$(i i) \rightarrow(i):$ The condition (i) is equivalent to $|t s|=|s t|$, where we have $|c|=\left(c^{*} c\right)^{\frac{1}{2}}$. Thus $t s$ is normal. It follows that

$$
\operatorname{ker}(t s)=\operatorname{ker}(s t)=\operatorname{ker}(|t s|)=\operatorname{ker}(|s t|) .
$$

Now let $Q$ denotes the associated projection. By theorem 1.4 and 1.5 we have the ranges of these four operators are closed and all coincide. Since we have

$$
s t(E)=\overline{s t(E)}=\overline{(t s)^{*}(E)}=\overline{(t s)^{*}(t s)(E)}=\overline{t s^{2} t E}=\overline{(s t)^{*} E}=t s(E) .
$$

Let $P$ denotes the associated projection to $\operatorname{ran}(t s)=\operatorname{ran}(s t)=\operatorname{ran}(|t s|)=$ 
$\operatorname{ran}(|s t|)$.

Hence $P+Q=I$. By polar decomposition theorem there are partial isometries $v, w$ such that $t s=v|t s|$, st $=w|s t|$. Note that $v v^{*}=v^{*} v=w w^{*}=w^{*} w=$ $P$. Since $P$ is a projection in $L(E)$ associated to $\operatorname{ran}(\operatorname{ts})=\operatorname{ran}(v)=\operatorname{ker}(v)^{\perp}$ hence if $x \in \operatorname{ker}(v)^{\perp}$ then $\left\langle v^{*} v(x), x\right\rangle=|v(x)|^{2}=|x|^{2}=\langle x, x\rangle=\langle P(x), x\rangle$ and if $x \in \operatorname{ker}(v)$ then $\left\langle v^{*} v(x), x\right\rangle=0=\langle P(x), x\rangle$. Hence by polarization identity $v^{*} v=P$.

Similarly $v v^{*}=w^{*} w=w w^{*}=P$. It is easily verified that $|t s| P=|t s|$. From this it obtains that $v, v^{*}, w$ and $w^{*}$ commute with $|t s|$. By assumption it follows that $|t s|=|s t|$. It yields that

$$
t s=v|t s|=|s t| w^{*}=w^{*}|t s|
$$

and since the range of $|t s|$ is dense in $P(E)$ it follows that $v=w^{*}$ and $v^{*}=w$. Now we have

$$
t s=v|t s|=v|s t|=v w^{*} s t=v^{2} s t .
$$

We can extend $\left.v^{2}\right|_{P(E)}$ to a unitary map $u=\left.v^{2}\right|_{P(E)} \oplus Q$.

\section{References:}

[1] E. C. Lance, Hilbert $C^{*}-$ Module, Cambridge University Press, London, 1995 .

[2] Zhang Lunchuan, The Characterization of Bounded Generalized Inverse Module Maps and Application to $C^{*}$-Algebras Factor Decomposition. preprint. [3] W. Rudin, Principles of Mathematical Analysis, McGraw-Hill, New York, 1976.

Received: May 2, 2006 